\title{
Accessing relevant information during problem solving: Time constraints on search in the problem space
}

\author{
EDWARD M. BOWDEN \\ University of Oregon, Eugene, Oregon
}

\begin{abstract}
An experiment examines the effect of time constraints on the ability of subjects to utilize relevant information during problem solving. Previous studies found that potentially relevant information was not effectively utilized unless subjects were explicitly informed of the information's relevance to the problem. The present study demonstrates that subjects who are not informed of the relation between relevant information and the problem are still able to access the information if allowed adequate time. These findings are interpreted within a model of problem solving that postulates search in a problem space as fundamental to the problem solving process.
\end{abstract}

Experimental studies of problem solving have suggested that people do not make effective use of relevant information unless explicitly informed of the information's relevance to the problem's solution (Gick \& Holyoak, 1980; Perfetto, Bransford, \& Franks, 1983; Weisberg, DiCammillo, \& Phillips, 1978). However, this conclusion seems to be in conflict with everyday experience. In real life situations, problem solvers are rarely explicitly told what information might be relevant to a problem's solution. Yet, people are able to solve many of the problems they encounter. The present study reconciles some of the disparity between previous experiments and our everyday experiences.

In a study by Weisberg et al. (1978), subjects first learned a list of word pairs according to a standard pairedassociate procedure. All word pairs consisted of highly associated words, such as table-chair, except the critical pair, candle-box. The list was learned to a criterion of two consecutive trials with perfect recall. Subjects next attempted to solve Duncker's (1945) candle problem: Use only a box of nails, a book of matches, a small hammer, and a candle to attach a candle to the wall so that it will burn properly. The preferred solution is to empty the box of nails and attach it to the wall so that it will serve as a platform for the candle.

Weisberg et al. (1978) manipulated the subjects' awareness of the relation between the critical word pair and the problem across three conditions. In one condition, subjects were informed that one of the word pairs learned earlier might be useful in solving the problem; subjects

I would like to thank Douglas Hintzman, Ray Hyman, and Morton Gernsbacher for their helpful advice during the course of this experiment, and for comments on earlier drafts of this paper. Thanks also go to Myron Rothbart for comments that helped clarify the results and to J. J. Franks and an anonymous reviewer for their suggestions. Please address correspondence to Edward M. Bowden, Department of Psychology, University of Oregon, Eugene, OR 97403. in a second condition were not so informed. Subjects in a third condition served as a baseline condition; they learned a control list that contained the irrelevant word pair candle-paper rather than candle-box. Weisberg et al. found that the candle-box pair was effective in cuing the preferred solution only when subjects were explicitly informed that the word pairs were relevant to the problem's solution.

In a second experiment, subjects learned the same paired-associate list; however, the word pairs were replaced with object pairs. Again, subjects attempted to solve Duncker's (1945) candle problem. The results from this second experiment were essentially identical to those obtained in the first: The candle-box pair was effective in cuing the preferred solution only when subjects were informed of its relevance to the solution.

Perfetto et al. (1983) reported similar results. In their experiments, subjects first rated the truthfulness of 14 sentences. After a short delay, subjects attempted to solve a series of riddles. The sentences rated earlier served as blatant clues to the riddles' solutions. For example, one riddle was "A man who lived in a small town in the U.S. married 20 different women of the same town. All are still living and he has never divorced one of them. Yet, he has broken no law." The clue sentence for this riddle was "A minister marries several people each week."

As in the Weisberg et al. (1978) experiment, Perfetto et al.'s (1983) subjects were divided into three conditions. One group of subjects was informed of the relation between the clue sentences and the riddles; a second group was not so informed; a third, baseline, group did not do the sentence rating task. And, like Weisberg et al., Perfetto et al. found that the clue sentences were effective in cuing the riddles' solutions only when subjects were explicitly informed that the sentences were relevant to the riddles' solutions.

Both of these studies seem to indicate that individuals do not make effective use of potentially relevant infor- 
ination during problem solving unless explicitly directid to do so. Such results pose serious difficulties for problem .olving in real-life situations. In most ordinary situations. problem solvers do not have the privilege of being told which information they possess is relevant to a problem's solution. Yet. people are able to solve most problems in real life. However, there might be other variables that influence the likelihood of subjects' using relevant information, even in the absence of explicit directions. One such variable might be the amount of time that the individual has to work on the problem. In the previous studies, subjects might not have used potentially relevant information because of time constraints, rather than because it was necessary to be explicitly informed of the information's relevance.

Weisberg et al. (1978) allowed their subjects to work on the candle problem until either the box was used in a solution or $2 \mathrm{~min}$ had elapsed since the subjects had overtly attempted a solution. It is impossible to precisely determine the mean time Weisberg et al. 's subjects required to produce the preferred solution, but the data suggest that informed subjects required less time than uninformed subjects. Nearly half of the informed subjects offered the preferred solution as their first solution (within $2 \mathrm{~min}$ ), whereas only one uninformed subject did so. In other studies employing Duncker's (1945) candle problem, the average solution time, in conditions similar to the uninformed condition of Weisberg et al., was around 11 min (Glucksberg, 1964; Glucksberg \& Weisberg, 1966). In fact, half the subjects in Glucksberg's (1964) study failed to produce the preferred solution in $15 \mathrm{~min}$ (the total time allowed). This strongly suggests that allowing only $2 \mathrm{~min}$ after the last overtly attempted solution might not be sufficient.

Perfetto et al. (1983) allowed their subjects only $40 \mathrm{sec}$ to solve each riddle. Assuming that at least $25 \%$ of this time was nceded to read and understand the riddle, and at least another $25 \%$ to write the solution, only $20 \mathrm{sec}$ remained for the actual process of problem solving. For an informed subject, 20 sec might be adequate; however. uninformed subjects might require additional time.

Perhaps if Weisberg et al. (1978) had allowed subjects more than $\mathbf{2}$ min after their last attempted solution, or if Perfetto et al.'s (1983) subjects had been allowed more than $\mathbf{4 0} \mathrm{sec}$ per riddle, then the uninformed subjects in each study might have made effective use of the relevant information to solve their respective problems. An initial experiment was conducted to test such a possibility.

One hundred twenty-nine subjects were tested with a procedure similar to that employed by Perfetto et al. (1983). The critical difference was the amount of time that subjects were allowed to work on each riddle: Perfetto et al. allowed subjects $\mathbf{4 0 ~} \mathrm{sec}$, whereas the subjects in the present experiment were allowed $2 \mathrm{~min}$. The results of this experiment differed from those reported by Weisberg et al. (1978) and Perfetto et al. The mean proportion of solutions that were congruent with the clue seniences were $0.62(S D=0.242), 0.62(S D=0.204)$. and
0.38 ISD $=0.135$, for the informed. uninforned. and baseline conditions, respectively. A one-way analysis of variance revealeci a significant main effect of experimental condition $[F(2.126)=17.085, p<.001]$. Because mean proportions of congruent solutions were identical for informed and uninlormed conditions. no pairwise comparisons were performed.

The above results demonstrate that subjects who were not explicitly informed of the relevance of the clue sentences produced congruent solutions as frequently as did subjects who were in the informed condition. Simply allowing subjects more time to work on the problems seems sufficient to compensate for any advantage of being told which information is relevant to the problems' solutions. But why is this true? How does increasing the amount of time that uninformed subjects have available to work on the problem improve their performance to the level of informed subjects? One model of problem solving that is able to account for this improvement with additional time was advanced by Newell and Simon (1972).

Newell and Simon (1972) proposed that problem solving involves a search in a "problem space" that contains not only the correct solution, but all possible solutions that might be considered. Informing subjects of the clues relevance to the problems' solutions could potentially constrain the subjects' search of the problem space by directing the search. This would increase the probability of finding the relevant information in a limited amount of time over the probability for an uninformed group whose search would not be so constrained.

As an analogy, consider the problem space as a dresser drawer filled with socks. The problem one is confronted with is to find one's favorite pair. If one is told approximately where to look for that pair of socks, for example, the top right-hand corner of the drawer, one should be able to locate the socks more quickly than if one has to search through the entire drawer. However, if one is intent upon finding the socks and they are in fact in the drawer, one eventually will locate them even without instructions concerning where to look. Yet, this will depend on how long one can search the drawer. Based on this model, one prediction is that the performance of uninformed subjects could be increased to the level of the performance of informed subjects simply by allowing them more time to work on the problems. The model also predicts that the performance of the baseline subjects should improve with additional time, but that their performance will remain inferior to that of the informed and uninformed subjects because they not only do not know where to look, but may not even have the correct pair of socks in their drawer. These predictions were verified in the above experiment.

Another prediction based on this model is that informed subjects will produce the majority of their congruent solutions early in the solution period because the effective "problem space" is relatively small, whereas uninformed subjects will produce the majority of their congruent solutions later in the solution period. To the 
extent that baseline subjects are able to solve the problems, their solutions should also occur later than those of the informed subjects. These predictions were tested in the following experiment.

\section{METHOD}

\section{Subjects}

The subjects were 60 undergraduate psychology students at the University of Oregon who participated for course credit.

\section{Materials}

The materials were 10 experimental riddles adapted from Gardner (1978). In addition, 10 sentences were constructed. Each sentence clearly cued the solution to one of the 10 riddles. These materials were similar, although not identical, to those used by Perfetto et al. (1983), and are included in the Appendix. Five filler sentences of the same style, but unrelated to the riddles, were also used.

Three additional riddles were taken from Gardner (1978). The solutions to these riddles were not cued by the sentences. The purpose of these riddles was to further separate the sentence-acquisition task from the problem-solving task. These, in addition to the 10 experimental riddles, comprised a packet of 13 riddles. Each page of the packet contained a single riddle; the three foils were the first three riddles, followed by the experimental riddles in a single randomly selected order.

\section{Procedure}

Twenty subjects were tested in each condition. The subjects were tested in groups of 2 to 10 . The subjects first rated the truthfulness of the 15 clue sentences, and after a 5-min interval during which the subjects performed an unrelated task, the riddle packet was presented. The subjects were allowed 2 min to work on each riddle. They were not allowed to turn to the next riddle until the full $2 \mathrm{~min}$ had elapsed, and they were instructed to write any solutions that they felt were correct. In addition, the subjects were instructed to draw a line across the paper immediately on the experimenter's command. The command was given at 20 -sec intervals during the 2 -min periods. The subjects were instructed to write any solution they might produce beneath the most recent line and if interrupted in the middle of a solution, to continue it beneath the line. This allowed the time of each solution to be roughly identified.

There were three experimental conditions. After the initial sentence-acquistion task, the informed subjects were told that the sentences they had rated earlier would be helpful in solving most of the riddles. The uninformed subjects did not receive these instructions. The subjects in the baseline condition worked on the riddles without having seen the clue sentences.

Following the problem-solving task, all subjects were asked to fill out a questionnaire designed to assess the degree to which informed and uninformed subjects were aware of the relation between the clue sentences and the riddles. The subjects were also asked to indicate which, if any, riddles they had seen prior to the experiment, and to answer several general questions regarding their problem-solving strategies.

The data from the subjects who admitted to having previously seen two or more of the experimental riddles were excluded from analysis, and additional subjects were tested. Data from two subjects in the uninformed group and one subject in the baseline group were excluded.

\section{RESULTS}

The purpose of the present experiment was twofold: to replicate the results of the author's earlier study in which subjects were allowed $2 \mathrm{~min}$ to solve each riddle; and to investigate potential differences in the time at which informed and uninformed subjects produced congruent solutions. The 2-min solution periods were divided into sixths according to the lines drawn across the response forms, and the cumulative number of congruent solutions was recorded for each interval. These data are presented in Figure 1.

Mean proportions of congruent solutions produced at the end of the 2-min period were $0.58(\mathrm{SD}=0.263), 0.54$ $(\mathrm{SD}=0.258)$, and $0.36(\mathrm{SD}=0.193)$ for the informed, uninformed, and baseline groups, respectively. A one-way analysis of variance yielded a significant main effect of experimental condition $[F(2,57)=4.92, p<.01]$. Pairwise comparisons revealed no significant difference between the performances of informed and uninformed subjects $[\mathrm{t}(38)<1.0, \mathrm{p}>.05]$. However, the performance of both informed $[\mathrm{t}(38)=2.96, \mathrm{p}=.004]$ and uninformed subjects $[\mathrm{t}(38)=2.37, \mathrm{p}=.02]$ was reliably superior to that of baseline subjects. These results replicate those of the author's previous experiment. The subjects who were not explicitly informed of the relevance of the clue sentences produced congruent solutions as frequently as the subjects who were so informed. Allowing uninformed subjects additional time to work on the riddles appears to be sufficient to compensate for informed subjects' advantage of being told what information is relevant to the riddles' solutions.

However, it is possible that the performance of uninformed subjects after $2 \mathrm{~min}$ is equal to the performance of informed subjects simply because their performance is equal throughout the 2 -min period. Therefore, overall analyses of variance and pairwise comparisons were performed to investigate the relative performance of each group at the various intervals. These analyses of the data at each of the six time intervals revealed that the performance of uninformed subjects improved with tirne, and that their performance did not simply parallel that of the

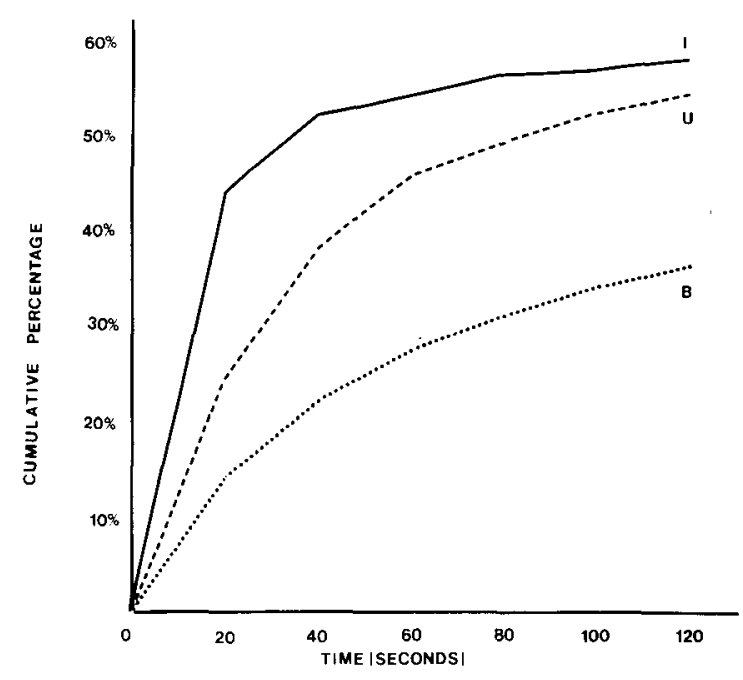

Figure 1. Cumulative percentage of congruent solutions at six intervals within the 2 -min solution period. $I=$ informed, $U=$ uninformed, and $B=$ baseline. 
informed subjects. Mean proportions of congruent solutions produced during the first interval were 0.44 (SD $=$ $0.262), 0.24(\mathrm{SD}=0.170)$, and $0.14(\mathrm{SD}=0.093)$ for the informed, uninformed, and baseline conditions, respectively. A one-way analysis of variance revealed a significant main effect of experimental condition $[F(2,57)$ $=13.09, \mathrm{p}<.0001]$. Pairwise comparisons yielded results remarkably similar to those reported by Perfetto et al. (1983) after $40 \mathrm{sec}$. Performance of informed subjects was superior to the performance of both uninformed $[\mathrm{t}(38)=3.28, \mathrm{p}=.002]$ and baseline $[\mathrm{t}(38)=5.04$, $\mathrm{p}=.001]$ subjects, whereas performance of uninformed subjects did not differ reliably from that of baseline subjects $[\mathrm{t}(38)=1.76, \mathrm{p}>.05]$. This suggests that the relatively poorer performance of uninformed subjects in the experiment of Perfetto et al. (1983) was due to their inability to access the relevant information as rapidly as informed subjects. ${ }^{1}$

Further analyses were performed to discover at what point in time the performance of the uninformed and baseline subjects first differed reliably, and at what point the performance of the uninformed and informed subjects no longer differed. These analyses revealed that the informed, uninformed, and baseline groups all differed reliably by the end of $40 \mathrm{sec}$. Mean proportions of congruent solutions produced by the end of the second interval were $0.52(S D=0.273), 0.37(S D=0.221)$, and $0.21(S D=0.157)$ for the informed, uninformed, and baseline groups, respectively. The performance of the informed subjects was superior to the performance of both the uninformed $[\mathrm{t}(38)=2.17, \mathrm{p}=.036]$ and the baseline subjects [t $(38)=4.68, p<.001]$. The performance of the uninformed subjects differed reliably from the performance of the baseline subjects [ $\mathrm{t}(38)=2.49, \mathrm{p}=$ $.018]$. At the end of $60 \mathrm{sec}$, mean proportions of congruent solutions were $0.54(\mathrm{SD}=0.256), 0.46(\mathrm{SD}=$ $0.254)$, and $0.26(S D=0.188)$. The performance of the informed and uninformed subjects no longer differed reliably $[\mathrm{t}(38)=0.99, \mathrm{p}=.328]$, whereas the performance of both groups was superior to the performance of the baseline subjects $[\mathrm{t}(38)=3.94, \mathrm{p}<.001$, for informed vs. baseline, and $t(38)=2.83, p=.008$, for uninformed vs. baseline].
The precise times at which the performance of the uninformed subjects differed reliably from the performance of the baseline subjects and no longer differed reliably from the performance of informed subjects have no special significance. These times would be expected to change with changes in the difficulty of the problems and with how blatantly the clues suggest the answers. The results at these intervals demonstrate the differential effect of time on performance in the three clue conditions. The subjects in the informed condition produced $76 \%$ of their total solutions during the first 20 -sec interval, whereas the subjects in the uninformed and baseline conditions produced $46 \%$ and $39 \%$, respectively, of their total solutions in that same interval. Percentages and mean time intervals of congruent solutions are presented by riddle and by condition in Table 1. These results further suggest that, with the passage of time, the subjects in the uninformed condition made a transition from being in a state similar to that of the baseline subjects (in which little relevant information was accessed) to being in a state similar to that of the informed subjects (in which relevant information was spontaneously accessed). The rate of this transition is illustrated in Figure 1.

One possible explanation for this apparent transition is that the uninformed subjects "caught on" to the relation between the clue sentences and the experimental riddles at some time during the experiment, and thus were no longer truly uninformed. To determine whether this explanation was likely, the 10 experimental riddles were divided into first 5 riddles and last 5 riddles in presentation order. The time interval in which a congruent solution was produced was then recorded for each of the riddles. Solutions produced during the first interval were assigned a score of 1 , solutions produced during the second interval were assigned a 2 , and so on, for all six intervals. If a riddle was unsolved at the end of the 2-min period, a score of 7 was assigned. Mean time intervals for the first 5 riddles and last 5 riddles were calculated for each subject. These mean time intervals were 3.52 $(\mathrm{SD}=1.11)$ for the first 5 riddles and $4.08(\mathrm{SD}=2.19)$ for the last 5 riddles for the informed subjects, 3.86 (SD $=1.41)$ and $4.94(\mathrm{SD}=1.76)$ for the uninformed subjects, and $4.85(\mathrm{SD}=1.35)$ and $5.83(\mathrm{SD}=0.88)$ for

Table 1

Percentage of Solutions, by Riddle, and Mean Time Interval (1-7) in Which Solutions Were Produced

\begin{tabular}{|c|c|c|c|c|c|c|c|c|c|}
\hline \multirow[b]{2}{*}{$\begin{array}{c}\text { Riddle } \\
\text { Position }\end{array}$} & \multicolumn{3}{|c|}{ Informed } & \multicolumn{3}{|c|}{ Uninformed } & \multicolumn{3}{|c|}{ Baseline } \\
\hline & $\begin{array}{l}\text { \% Subjects } \\
\text { Solving }\end{array}$ & $\begin{array}{l}\text { Mean } \\
\text { Time } \\
\end{array}$ & SD & $\begin{array}{c}\% \text { Subjects } \\
\text { Solving }\end{array}$ & $\begin{array}{l}\text { Mean } \\
\text { Time }\end{array}$ & SD & $\begin{array}{c}\% \text { Subjects } \\
\text { Solving }\end{array}$ & $\begin{array}{l}\text { Mean } \\
\text { Time }\end{array}$ & $\mathrm{SD}$ \\
\hline 1 & 80 & 1.56 & 1.41 & 85 & 1.29 & 0.59 & 35 & 2.14 & 1.46 \\
\hline 2 & 85 & 1.29 & 0.85 & 80 & 1.94 & 1.24 & 65 & 2.92 & 1.80 \\
\hline 3 & 100 & 1.20 & 0.41 & 80 & 1.63 & 0.72 & 85 & 1.71 & 1.53 \\
\hline 4 & 30 & 1.17 & 0.41 & 35 & 1.57 & 0.79 & 30 & 1.83 & 1.33 \\
\hline 5 & 20 & 3,75 & 0.96 & 15 & 3.00 & 2.65 & 15 & 4.67 & 1.53 \\
\hline 6 & 50 & 1.70 & 1.25 & 45 & 2.67 & 1.80 & 0 & 7.00 & 0.00 \\
\hline 7 & 60 & 1.50 & 0.80 & 55 & 2.64 & 1.57 & 40 & 3.00 & 1.41 \\
\hline 8 & 60 & 1.25 & 0.62 & 40 & 2.25 & 0.46 & 20 & 2.00 & 0.82 \\
\hline 9 & 45 & 1.44 & 0.53 & 50 & 1.90 & 1.29 & 30 & 3.33 & 1.21 \\
\hline 10 & 50 & 1.60 & 1.56 & 50 & 3.00 & 1.41 & 40 & 2.25 & 0.89 \\
\hline
\end{tabular}


the baseline subjects. A 3 (experimental condition) $\times 2$ (riddle position) analysis of variance yielded significant main effects of experimental condition $[\mathrm{F}(2,57)=7.22$, $\mathrm{p}=.001]$ and riddle position $[\mathrm{F}(1,57)=18.60, \mathrm{p}<$ $.001]$, and a nonsignificant interaction effect $[F(2,57)<$ $1.0, \mathrm{p}>.05]$.

The main effect of experimental condition simply shows again that there was a difference in the average amounts of time required by the informed, uninformed, and baseline subjects to produce congruent solutions. The main effect of riddle position suggests that some of the experimental riddles were more difficult than others, in that they required more time to be solved or were solved less often. This result is to be expected, since the riddles certainly varied in difficulty and their presentation order was the same for all subjects. The interesting result is the lack of a significant interaction between experimental condition and riddle position. If uninformed subjects "caught on" to the relation between the clue sentences and the experimental riddles, one would expect the change in their first-half to second-half solution times to be more positive (or less negative) than the changes of the informed and baseline subjects. The lack of an interaction suggests that the differences among the three groups remained essentially the same across riddles. Thus, there is no evidence that the uninformed subjects improved because they became "informed" over the course of the experiment.

Further evidence suggesting that uninformed subjects remained less aware of the relation between the clue sentences and the riddles' solutions comes from the postexperiment questionnaire. All 20 informed subjects indicated that they thought of the clue sentences while attempting to solve the riddles, whereas 14 of 20 uninformed subjects indicated the same $\left[\chi^{2}=7.059\right.$, $p<.05]$. This suggests that although by the conclusion of the experiment many uninformed subjects had some knowledge of the relation between the clue sentences and the riddles, they remained less aware of the relation than the informed subjects. There is evidence that people are frequently unable to report accurately the influence of stimuli on their responses (Nisbett \& Wilson, 1977; see Duncker, 1945, and Maier, 1931, for instances directly related to problem solving). Subjects appear to be willing to report a stimulus as being influential if it seems to be a plausible cause of the response. If the stimulus does not seem to be a plausible cause of the response, subjects report it as noninfluential. Clearly, the clue sentences are a plausible cause of the subjects' solutions and thus are likely to be reported, in retrospect, as being influential. This does not necessarily mean that subjects are accurately reporting their own awareness of their use of clues during problem solving.

\section{DISCUSSION}

Perfetto et al. (1983) suggested that the failure of uninformed subjects to utilize relevant information during problem solving found in previous research (Gick \& Holy- oak, 1980; Weisberg et al., 1978) might be explained in two ways: (1) Subjects might spontaneously retrieve the appropriate information, but fail to recognize its relevance to the problem, or (2) subjects might simply fail to spontaneously retrieve the information at all. Their results seemed to rule out the first explanation while lending support for the second. However, the results of the present experiments suggest a third possible explanation. Uninformed subjects in previous studies might have failed to retrieve the appropriate information because of time constraints imposed by the experiments. The present study demonstrates that uninformed subjects can and do utilize previously acquired information if they are allowed adequate time.

Obviously, the amount of time that would be considered adequate should depend on the difficulty of the problem and how blatantly the clues suggest the solution. It is also obvious that increasing the time available to work on a problem will increase the likelihood of subjects' producing correct solutions in general. This is demonstrated by the improved performance of baseline subjects with increased time. Improvement by the baseline subjects probably reflects access to relevant information acquired outside the experimental situation. In some sense, almost all persons in our culture have information relevant to at least some of the riddles available in memory. For both informed and uninformed subjects, this information is made more accessible by its recent presentation in the form of the clue sentences. Informed subjects have the additional advantage of being explicitly informed of the information's relation to the problem.

The present experiment produced two major findings. First, uninformed subjects were able to access and utilize relevant information when the time available to work on the riddles was increased from $40 \mathrm{sec}$ to $2 \mathrm{~min}$. Second, informed subjects produced the majority of their congruent solutions early in the 2-min period, whereas uninformed subjects produced the majority of their congruent solutions later in the period. These findings suggest that informing subjects of the relation between relevant information and a problem's solution facilitates problem solving by allowing them to access the information more rapidly.

Earlier in this paper it was suggested that the process of search is fundamental to the problem-solving process. Problem solving was said to take place by a search in a problem space - that is, by considering one knowledge state after another until a desired knowledge state is reached and the problem is solved (Newell \& Simon, 1972). This model of the problem-solving process suggests that when presented with a problem, the subject uses the problem as a retrieval cue to search memory for any information that will lead to a solution. The uninformed subjects in the present experiment appear to have made greater access to the clue sentences within $40 \mathrm{sec}$ than the uninformed subjects in the Perfetto et al. (1983) experiment. If the problems do serve as retrieval cues to search memory for relevant information, then the more 
similarity between the clues and the problems (the more blatantly they suggest the solutions), the less time subjects should require to access the information. It appears that the clue sentences in the present experiment had more exact words and phrases in common with the riddles than those used by Perfetto et al. This greater similarity might account for the more rapid access by uninformed subjects in the present experiment.

Newell and Simon (1972) have further suggested that when a subject searches the problem space, residence time in each particular knowledge state is on the order of seconds. This, combined with the assumption that the problem space contains not only the correct solution, but also all possible solutions that a person might consider, leads to the prediction that the more explicitly directed the search process, the fewer knowledge states the subject will consider, and the shorter the length of time that will be required to access relevant information. Explicitly informing subjects of the relation between information and a problem's solution facilitates problem solving by focusing, or directing, their search in the problem space.

In most real-world problem-solving situations, an essentially uninformed problem solver searches his or her memory for potentially relevant information. To have a realistic chance of solving problems under these circumstances, a person must not only have previously acquired information relevant to the problem's solution, but must also be able to access this information. Usually, the problem solver does not have the privilege of being told which of the information he or she possesses is relevant to the problem. Earlier research has suggested that this uninformed state can lead to serious difficulties in accessing information that would aid in the problem's solution (Gick \& Holyoak, 1980; Perfetto et al., 1983; Weisberg et al., 1978). The results of the present experiment suggest that the consequences of being uninformed are not as severe as prior studies have suggested. Being uninformed about the relation between relevant information and the problem does not appear to prevent solution of the problem. Rather, being explicitly informed of this relation facilitates problem solving by directing the search process, thereby decreasing the time necessary to access relevant information.

\section{REFERENCES}

DUNCKER, K. (1945). On problem-solving. Psychological Monographs, 58(5, Whole No. 270).

GARDNER, M. (1978). Aha! Insight. New York: Freeman.

Gick, M. L., \& HolyoAK, K. J. (1980). Analogical problem solving. Cognitive Psychology, 12, 306-355.

Glucksberg, S. (1964). Functional fixedness: Problem solving as a function of observing responses. Psychonomic Science, 1, 117-118.

Glucksberg, S., \& Weisberg, R. W. (1966). Verbal behavior and problem solving: Some effects of labelling in a functional fixedness problem. Journal of Experimental Psychology, 71, 659-664.

MaIER, N. R. F. (1931). The solution of a problem and its appearance in consciousness. Journal of Comparative Psychology, 12, 181-194.

NeWELl, A., \& SimON, H. A. (1972). Human problem-solving. Englewood Cliffs, NJ: Prentice-Hall.

NisbeTt, R. E., \& WiLson, T. D. (1977). Telling more than we can know: Verbal reports on mental processes. Psychological Review, 84, 231-259.

Perfetto, G. A., Bransford, J. D., \& Franks, J. J. (1983). Constraints on access in a problem solving context. Memory \& Cognition, 11, 24-31.

Weisberg, R., DiCamillo, M., \& Phillips, D. (1978). Transferring old associations to new situations: A nonautomatic process. Journal of Verbal Learning and Verbal Behavior, 17, 219-228.

\section{NOTES}

1. Inspection of the data at each time interval (see Figure 1) clearly shows an interaction between time and condition. It might seem more straightforward to simply test this interaction. The interaction was tested in a number of ways, each of which was not totally satisfactory due to the dependence of performance at each interval on performance at the preceding intervals. All of these tests yielded highly significant interactions. It therefore appears safe to conclude that there is an interaction between time and condition, with uninformed subjects requiring more time to reach the same level of performance as informed subjects.

2 . This analysis is somewhat flawed in that the amount of time necessary to produce a congruent solution is confounded with whether a congruent solution is actually produced. Subjects who solved fewer riddles would have greater mean times to solution. However, there are several reasons to believe that the conclusions drawn remain valid despite this confounding. First, this confounding would be a serious problem only if there were a difference between informed and uninformed subjects in total number of riddles solved. The difference is minimal. Second, if a subject failed to produce a solution within a 2-minute period, it seems reasonable to assume that he or she required more time, and to assign the next greater time value to that riddle. If time values are assigned to all subjects in the same manner, it is extremely unlikely that the precise circumstances necessary to conceal an interaction would exist. Third, when mean time intervals are computed for only congruent solutions, the pattern of results remains the same (see Table 1). Uninformed subjects, on the average, required more time to produce congruent solutions than informed subjects. Finally, a number of other tests were performed, all of which yielded nonsignificant interactions between riddle position and condition. Although time and solution production are confounded in the analysis presented, it seems reasonable to conclude that the performance of uninformed subjects cannot be explained simply by saying that they became informed during the course of the experiment.

\section{APPENDIX \\ Materials Used in the Experiment}

1. A woman walked for twenty minutes on the surface of a lake without sinking into the water. She was not using any form of flotation device, such as a boat or a raft. How did she manage to do it?

A person can walk on a lake when it is frozen

2. One night a man was reading a book when his wife turned off the lights. Even though the room was pitch dark he went right on reading. How could he do this?

A blind person can read a braille book in the dark.

3. A man was caught in the rain with no hat or umbrella. There was nothing over his head and his clothes got soaked, but not a hair on his head got wet. How is this possible?

After taking a shower a bald man does not have to dry his hair.

4. If you had only one match and you wanted to light a kerosene lamp, an oil heater, and a wood burning stove, which would you light first?

You must first light a match before you can light a fire. 
5. A wine bottle is half filled and corked. How can you drink all of the wine without breaking the bottle or removing the cork from the bottle?

A cork can be opened by pulling it out or by pushing it in.

6. You have four nines $(9,9,9,9)$. Using any arithmetical processes (addition, subtraction, multiplication, division) arrange the four nines so that the total is 100 .

Nine divided by nine plus ninety-nine is equal to one hundred.

7. A man who lived in a small town in the United States married 20 different women of the same town. All are still living and he has never divorced any of them. Yet, he has broken no law. Can you explain?

A minister can marry several different women and men every month.
8. Uriah Fuller, the famous superpsychic, can tell you the score of any baseball game before it starts. What is his secret?

The score of any baseball game, before it starts, is nothing to nothing.

9. Why are 1977 dollar bills worth more than 1976 dollar bills?

1,966 dollar bills are worth more than 1,965 dollar bills.

10. A young boy turned off the light in his bedroom and managed to get into bed before the room was dark. If the bed is ten feet from the light switch and he used no wires, strings, or other contraptions to turn off the light, how did he do it?

A bedroom is not usually dark during the daytime.

(Manuscript received December 19, 1984; revision accepted for publication March 25, 1985.) 